\title{
Coalitions of End-use Customers in Retail Electricity Markets: A Real-World Case Study Involving Five Schools for Children
}

\author{
Hugo Algarvio, ${ }^{1,2, \star}$ Fernando Lopes ${ }^{2, \star}$ and João Santana ${ }^{1}$ \\ 1 Instituto Superior Técnico, Universidade de Lisboa, INESC-ID, Lisbon, Portugal \\ \{hugo.algarvio, jsantana\}@tecnico.ulisboa.pt \\ 2 LNEG-National Research Institute, Est. Paço do Lumiar 22, Lisbon, Portugal \\ fernando.lopes@lneg.pt
}

\begin{abstract}
The key mechanisms for purchasing and selling electrical energy include electricity pools and bilateral contracts. This article is devoted to bilateral contracting, which is modeled as a negotiation process involving an iterative exchange of offers and counter-offers. It focuses on coalitions of end-use consumers and describes a case study involving five schools for children located in England. The schools decide to ally into a coalition to strengthen their bargaining positions and, hopefully, to obtain better tariffs. To this end, they rely on a coordinator agent, who is defined from the group of five schools, by selecting either the "most powerful" school or the "best negotiator" school. The coordinator takes decisions according to either a "majority" rule, a "consensus" rule, or an "unanimity" rule. The simulations are performed with an agentbased system, called MATREM (for Multi-agent TRading in Electricity Markets). Although preliminary, the results suggest that coalition formation and management is beneficial to end-use customers, since the price agreed in the new forward contracts is more favorable to these agents, particularly when the coordinator is the "best negotiator" agent and considers the "unanimity" decision rule.
\end{abstract}

Keywords: Electricity markets; bilateral contracts; customer coalitions; trading strategies, decision rules, MATREM system.

\section{Introduction}

The liberalization of electricity markets (EMs) has led to the establishment of a wholesale market, where competing generators offer their energy to retailers, and a retail market, in which retailers ensure delivery to end-use customers $[1,2]$. Because of transaction costs, only largest consumers choose to purchase energy directly on the wholesale market. Most small and medium consumers purchase energy from retailers, who in turn buy it in the wholesale market [3].

\footnotetext{
* This work was supported by "Fundação para a Ciência e Tecnologia" with references UID/CEC/50021/2013 and PD/BD/105863/2014 (H. Algarvio).
} 